\title{
La temperatura de vernalización en bulbos reutilizados de lirio (Lilium sp.) afecta la distribución de materia seca y la producción de flor
}

\section{The vernalization temperature in reutilized lily (Lilium sp.) bulbs affects the distribution of dry matter and flower yield}

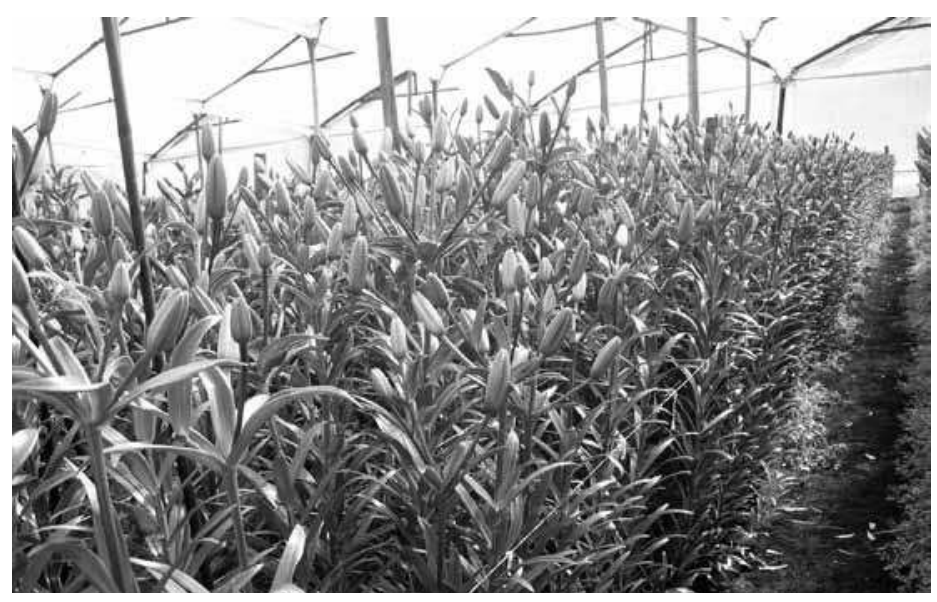

ORLANDO AUZAOUE-RODRÍGUEZ ${ }^{1}$

HELBER ENRIOUE BALAGUERA-LÓPEZ ${ }^{1,4}$

JAVIER GIOVANNI ÁLVAREZ-HERRERA ${ }^{2}$

GERHARD FISCHER ${ }^{3}$

\section{RESUMEN}

El lirio se propaga comercialmente mediante bulbos. Para los programas de siembra en Colombia, los bulbos deben ser importados, pero tienen un alto costo, lo cual reduce la rentabilidad del cultivo. No obstante, mediante la vernalización de los bulbos que quedan después del corte de la flor es posible obtener nuevas cosechas. Por tanto, el objetivo fue evaluar el efecto de diferentes temperaturas de vernalización en bulbos reutilizados de lirio del híbrido asiático Castello sobre la distribución de materia seca y calidad de la flor. Se utilizó un diseño completamente al azar con cuatro tratamientos correspondientes a tres temperaturas de vernalización $\left(-2^{\circ} \mathrm{C}, 3^{\circ} \mathrm{C}\right.$ y $14^{\circ} \mathrm{C}$ ) y un testigo comercial (bulbos importados), cada tratamiento tuvo tres repeticiones. Los bulbos fueron almacenados durante 8 semanas a las temperaturas mencionadas y luego fueron sembrados en campo. El testigo comercial generó mayor masa seca de flores, tallo, bulbo y total, y mejor calidad de flor, con la vernalización a $-2^{\circ} \mathrm{C}$ y $3^{\circ} \mathrm{C}$ se obtuvo una moderada masa seca de flores, tallo y total pero baja en bulbos, se presentó alta floración y buena calidad de flor de lirio, mientras que con $14^{\circ} \mathrm{C}$ no se presentó una removilización adecuada de las reservas del bulbo, lo que afectó negativamente el crecimiento y la floración. La vernalización de bulbos reutilizados de lirio es una práctica que potencialmente podría reemplazar la importación de bulbos, pero necesita más investigación para aumentar su eficiencia.

\footnotetext{
Facultad de Ciencias Agropecuarias, Grupo de Investigaciones Agrícolas, Universidad Pedagógica y Tecnológica de Colombia, Tunja (Colombia).

2 Facultad de Agronomía, Programa de Doctorado en Ciencias Agropecuarias, Área Agraria, Universidad Nacional de Colombia, Bogotá y Facultad de Ciencias Agropecuarias, Grupo de Investigaciones Agrícolas, Universidad Pedagógica y Tecnológica de Colombia, Tunja (Colombia).

3 Facultad de Agronomía, Departamento de Agronomía, Universidad Nacional de Colombia, Bogotá (Colombia).

4 Autor para correspondencia. enrique_balaguera@yahoo.com
} 


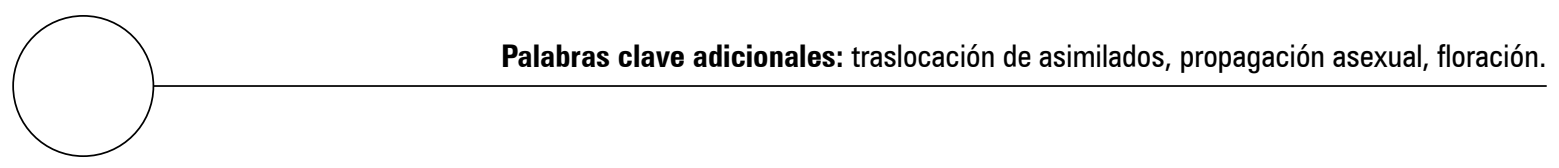

ABSTRACT

Lily is propagated commercially with bulbs. For planting programs in Colombia, these bulbs must be imported, but this is an expensive procedure, which reduces profitability. However, through vernalization of bulbs that remain after cutting the flower, it is possible to obtain a new crop cycle. Therefore, the objective was to evaluate the effect of different temperatures in the vernalization of reutilized lily bulbs of the Asian hybrid Castello on the distribution of dry matter and flower quality. A completely randomized design was used with four treatments for three vernalization temperatures $\left(-2^{\circ} \mathrm{C}, 3^{\circ} \mathrm{C}\right.$ y $\left.14^{\circ} \mathrm{C}\right)$ and a commercial control (imported bulbs), each treatment had three replications. The bulbs were stored for 8 weeks at the above-mentioned temperatures and then planted in the field. The commercial control produced greater dry mass for: flowers, stem, bulb and total; and better quality of flowers. With vernalization at $-2^{\circ} \mathrm{C}$ and $3^{\circ} \mathrm{C}$, a moderate dry mass for flowers, stems and bulbs was obtained, but with a low total dry mass; whereas lilies at these low temperatures bloomed abundantly and presented good quality flowers. At $14^{\circ} \mathrm{C}$, no adequate remobilization of reserves of the bulbs took place, which negatively affected growth and flowering. Vernalization of reutilized lily bulbs is a practice that potentially could replace bulb imports, but more research needs to increase its efficiency.

Additional keywords: assimilate translocation, asexual propagation, flowering.

Fecha de recepción: 09-08-2011

Aprobado para publicación: 21-11-2011

INTRODUCCIÓN

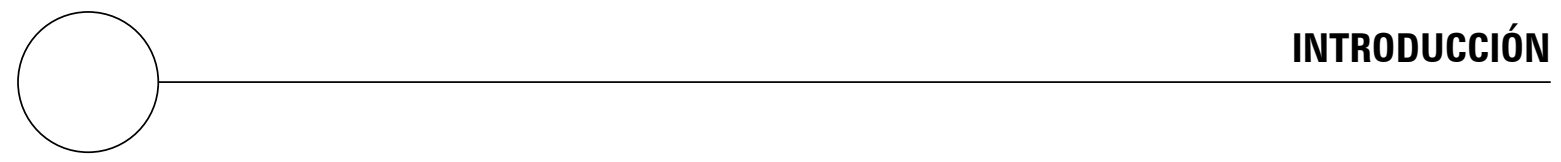

Las flores más vendidas en el mundo son, en primer lugar, las rosas, seguidas por los crisantemos, tercero los tulipanes, cuarto los claveles y en quinto lugar los lirios (Klock, 1997). Streck y Schuh (2005) afirman que el Lilium es uno de los géneros de bulbos de flores más importantes del mundo. El Lilium produce una flor de calidad muy apreciada por el consumidor, lo que asegura una buena demanda en el mercado. Colombia ha presentado un incremento representativo en las áreas de producción de lirio y en la comercialización, pasando de 2 ha en el año 2000 a 15 ha para el 2008 (Auzaque-Rodríguez et al., 2009). Este género puede comercializarse como flores cortadas frescas, plantas con flores en materas, jardines o paisajes (De Hertogh, 1996); a pesar de esto, Colombia solo produce flor de corte (Auzaque-Rodríguez et al., 2009). Según De Hertogh (1996), la importancia de los Lilium en el mercado mundial se debe en gran parte a la diversidad de las especies y al alto número de híbridos y cultivares comercialmente disponibles.

La vernalización es un término que describe la promoción de la floración después de la exposición a bajas temperaturas (Taiz y Zeiger, 2006; Sung y Amasino, 2004a). De acuerdo con Chouard (1960) y Sung y Amasino (2004a), la vernalización resulta en la adquisición o aceleración de la habilidad para florecer mediante un tratamiento con frío; después de la vernalización, las plantas no necesariamente inician la floración pero sí adquieren la competencia para hacerlo (Sung y Amasino, 2004a). El tratamiento con frío debe darse durante la fase de semilla hidratada o de planta joven (Azcón-Bieto y Talón, 2008). Según Sung y Amasino (2004a), se ha demostrado que el meristemo apical es el encargado de percibir 
el estímulo generado por las bajas temperaturas para que ocurra la floración. En el caso de los lirios, que también deben ser vernalizados para florecer (Streck y Schuh, 2005), las bajas temperaturas deben ser aplicadas a los bulbos (Lee et al., 1996).

La respuesta de las plantas a la vernalización está determinada por la temperatura y por la duración de las bajas temperaturas (Alberto et al., 2009; Streck y Schuh, 2005). Streck y Schuh (2005) afirman que plantas de lirio provenientes de bulbos sometidos a vernalización tuvieron una emergencia más rápida y uniforme, una disminución del ciclo de cultivo con una floración más precoz, comparadas con aquellas plantas que emergieron de bulbos no vernalizados. Se ha encontrado que la temperatura de vernalización es dependiente de cada especie y de cada cultivar, en Lilium rubellum se recomienda $4^{\circ} \mathrm{C}$ (Xu et al., 2006), para Lilium longiflorum 'Nellie White" $5^{\circ} \mathrm{C}$ (Kim et al., 2007), para híbridos orientales de lirio la temperatura de vernalización mínima es $1,5^{\circ} \mathrm{C}$ (Lee y Roh, 2001), la óptima $5^{\circ} \mathrm{C}$ y la máxima $21^{\circ} \mathrm{C}$ (Streck, 2002) y en Tulipa gesneriana L. 'Gander' los bulbos son vernalizados a $2^{\circ} \mathrm{C}$ (Inamoto et al., 2000).

A nivel comercial el lirio se propaga mediante bulbos, los cuales son muy costosos, y son el factor más importante que limita la expansión del cultivo en Colombia. Según Auzaque et al. (2009), en la Sabana de Bogotá, los bulbos para producción de flor de corte de lirio deben ser importados y mantenidos en cadena de frío hasta su siembra en campo. Este bulbo sembrado produce flor de calidad de exportación solamente en la primera cosecha, el bulbo que queda después del corte de la flor produce flores de baja calidad y generalmente es desechado, por tanto, el productor de lirio debe comprar nuevamente material para poder mantener sus programas de siembra, lo que incrementa los costos de producción y disminuye la rentabilidad del cultivo. Auzaque et al. (2009) sometieron bulbos reutilizados de lirio a tratamientos de vernalización artificial y encontraron que hubo una muy buena calidad de flor con el tratamiento de almacenamiento de bulbos reutilizados a $3^{\circ} \mathrm{C}$ durante 8 semanas, no obstante, la respuesta fue inferior a la del testigo comercial (Auzaque et al., 2009).

Además, se sabe que la vernalización afecta diferentes procesos fisiológicos como la distribución de masa seca, tal como se encontró en Lilium longiflorum (Kim et al., 2007) y Tulipa gesneriana L. (Inamoto et al., 2000), pero que en plantas para flor de corte de lirio aún no ha sido determinado y mucho menos con la utilización de bulbos reutilizados y bajo condiciones tropicales. Por tanto, el objetivo fue determinar el efecto la temperatura de vernalización de bulbos reutilizados de lirio sobre la distribución de materia seca y la calidad de flor, con el fin de generar estrategias que permitan disminuir la dependencia de la importación de bulbos para continuar con los programas de siembra y, de esta manera, aumentar la rentabilidad del cultivo de lirio.

\section{MATERIALES Y MÉTODOS}

La fase de campo se llevó a cabo en un invernadero de cubierta plástica, el cual se encuentra ubicado en el municipio de Madrid, Cundinamarca, a una altitud de $2.680 \mathrm{msnm}$. La temperatura ambiente media de la zona fue aproximadamente $14^{\circ} \mathrm{C}$, mientras que dentro del invernadero fue de $16,5^{\circ} \mathrm{C}$, con mínima y máxima promedio de 7 y $28^{\circ} \mathrm{C}$, respectivamente. La fase de laboratorio fue realizada en el Laboratorio de Fisiología Vegetal de la Facultad de Agronomía, Universidad Nacional de Colombia, sede Bogotá.

Se evaluaron cuatro tratamientos dispuestos en un diseño completamente al azar, dichos tratamientos correspondieron a tres temperaturas de vernalización de los bulbos $\left(-2^{\circ} \mathrm{C}, 3^{\circ} \mathrm{C}\right.$ y $\left.14^{\circ} \mathrm{C}\right)$ más un testigo comercial (bulbos importados vernalizados), estas temperaturas fueron seleccionadas a partir de los resultados obtenidos por Auzaque-Rodríguez et al. (2009). Cada trata- 
miento se replicó tres veces y cada una de las 12 unidades experimentales estuvo compuesta por 12 bulbos de lirio del híbrido asiático Castello.

Fue necesario efectuar dos siembras, en la primera se sembraron bulbos importados de calibre 14/16 (14 cm diámetro longitudinal/16 cm diámetro ecuatorial), de los cuales se obtuvieron los bulbos que se sometieron a vernalización artificial y que correspondieron a la segunda siembra. A los 105 días después de la siembra (dds) de los bulbos importados se obtuvo la floración y se procedió a la cosecha de la flor, teniendo la precaución de realizar el corte del tallo a aproximadamente $20 \mathrm{~cm}$ por encima del nivel del suelo, con el fin de que las hojas de la porción de tallo restante siguieran fotosintetizando $\mathrm{y}$, además, retraslocaran fotoasimilados al bulbo para que garantizaran un buen tamaño del mismo y una gran cantidad de reservas para la nueva planta. Estos bulbos fueron dejados en campo 6 semanas, tal como lo recomiendan Auzaque-Rodríguez et al. (2009). Pasado este tiempo, los bulbos fueron cosechados y se seleccionaron aquellos que tuvieron el calibre 12/14 para continuar con el experimento.

Los bulbos seleccionados para ser reutilizados se sometieron a los tratamientos de vernalización de la siguiente manera: para el almacenamiento a $\left(-2^{\circ} \mathrm{C}, 3^{\circ} \mathrm{C}\right.$ y $\left.14^{\circ} \mathrm{C}\right)$ y $3^{\circ} \mathrm{C}$ se utilizaron cuartos fríos, mientras que para los $14^{\circ} \mathrm{C}$ (temperatura ambiente promedio), los bulbos fueron dejados en un cuarto oscuro, fresco y seco a temperatura ambiente. El almacenamiento tuvo una duración de 8 semanas, tiempo con la respuesta más favorable para la vernalización artificial para lirio, obtenida por Auzaque-Rodríguez et al. (2009).

En las dos siembras, los bulbos fueron sembrados en cuadro a $12,5 \times 12,5 \mathrm{~cm}$ y a $10 \mathrm{~cm}$ de profundidad en camas de $0,8 \mathrm{~m}$ de ancho previamente preparadas. Las diferentes labores culturales propias del cultivo como fertilización, riego, tutorado y control fitosanitario, entre otras, se llevaron a cabo según lo recomendado para los cultivos comerciales de lirio de la Sabana de Bogotá (Auzaque, 2008). El corte de la flor se hizo cuando el primer o los dos primeros botones florales mostraron el color característico bien definido (en este caso color naranja), que fue entre la semana 14 a la 22, dependiendo de los tratamientos.

Las variables medidas en la cosecha fueron: masa seca de flores, hojas, tallo, bulbo, raíces y total, mediante medición directa en balanza electrónica de precisión $0,01 \mathrm{~g}$, después de someter las plantas en estufa a $75^{\circ} \mathrm{C}$ durante $24 \mathrm{~h}$; calidades comerciales: porcentaje de flores producidas pertenecientes a cada una de las calidades comerciales según la tabla 1.

Los datos obtenidos fueron sometidos a un análisis de varianza y a la prueba de comparación de medias de Tukey $(P \leq 0,05)$. También se realizó una prueba de correlaciones de Pearson y para el análisis de los datos se empleó el software SAS v. 8.1e (Cary, NC).

Tabla 1. Criterios para determinar las calidades comerciales de las flores de lirio, según Auzaque-Rodríguez et al. (2009).

\begin{tabular}{|l|c|l|l|l|}
\hline $\begin{array}{c}\text { Grado de } \\
\text { calidad }\end{array}$ & Longitud & $\begin{array}{c}N^{*} \text {. de botones } \\
\text { (longitud mínima de } \\
\text { botón } 2,5 \mathrm{~cm} \text { ) }\end{array}$ & $\begin{array}{c}\text { Torcedura tallo } \\
\text { Estado } \\
\text { fitosanitario }\end{array}$ \\
\hline Extra & $>65 \mathrm{~cm}$ & 40 más & No & Bueno \\
\hline Super 3 botones & $55-64 \mathrm{~cm}$ & 3 & No & Bueno \\
\hline Super 2 botones & $55-64 \mathrm{~cm}$ & 2 & Leve, máximo $10^{\circ}$ con relación al eje vertical & Bueno \\
\hline Nacional & $<55 \mathrm{~cm}$ & 1 o más & Leve, máximo $10^{\circ}$ con relación al eje vertical & Bueno \\
\hline
\end{tabular}

*La longitud se mide desde la base del tallo clasificado hasta el ápice del botón floral más alto. 


\section{RESULTADOS Y DISCUSIÓN}

\section{Masa seca de órganos}

Se presentaron diferencias estadísticas $(P \leq 0,01)$ en la masa seca total de la planta, de flores, hojas, tallo y bulbo, la masa seca de raíz no mostró diferencias significativas (figura 1). El testigo comercial generó mayor masa seca total, de flores, tallo y bulbo (figuras $1 \mathrm{~A}, \mathrm{~B}, \mathrm{D}$ y $\mathrm{E}$ ), mientras que con la vernalización a $3^{\circ} \mathrm{C}$ hubo mayor masa de hojas (figura $1 \mathrm{~F}$ ) y buena respuesta en la masa seca de tallo y total (figuras $1 \mathrm{~A}$ y D). Con la temperatura de $14^{\circ} \mathrm{C}$ se generó menor masa seca total, de flores, tallo y de las más altas en bulbo y raíz (figuras 1 A, B, D, E y F).

La respuesta favorable generada por los bulbos del testigo comercial, se debe, en gran medida, a la mayor cantidad de reservas disponibles que presentan en comparación con los bulbos reutilizados. Esta mayor cantidad de reservas junto con un tratamiento de vernalización adecuado garantizan una mayor conversión de carbohidratos a azúcares solubles y su consecuente transporte a la parte aérea (Moe, 1979); igualmente, esto hizo que la planta creciera más vigorosa y su eficiencia fotosintética aumentara, por lo que hubo mayor cantidad de asimilados que fueron traslocados eficientemente de las hojas a las flores, otra parte se quedó en el tallo y otra porción fue retraslocada al bulbo, pero las hojas no acumularon mayor cantidad de masa seca, poniendo en evidencia que la floración obtenida mediante vernalización estaría disminuyendo el crecimiento vegetativo. Con los tratamientos de $-2^{\circ}$ y $3^{\circ} \mathrm{C}$ se obtuvieron resultados similares, mientras que con los bulbos almacenados a $14^{\circ} \mathrm{C}$ se favoreció el crecimiento vegetativo, razón por la cual, la masa seca de flores fue baja, pero además, la falta de frío en este tratamiento al parecer no generó movilización suficiente de reservas del bulbo hacia la parte aérea, tal como lo reporta Cochrane et al. (1991), y por esto, el bulbo permaneció con una masa seca representativa, no obstante, el crecimiento no fue considerable y la masa seca total fue inferior que en los demás tratamientos.

Lo anterior lo corrobora el análisis de correlaciones, donde se encontró que la masa seca total es inversamente proporcional a la masa seca de hojas, pero es directamente proporcional con la masa seca de flores y de tallo; así mismo, la masa seca de hojas tiene una correlación negativa con la masa seca de bulbo, pero la masa seca total no tuvo correlación con la masa seca del bulbo (tabla 2). Al respecto, Inamoto et al. (2000) mencionan que la masa seca total está en función de la masa seca del bulbo, pues este último compite permanentemente con la masa seca aérea y hace menor la masa seca de la planta, también encontraron que la mayor masa seca de plantas de tulipán ocurrió con la vernalización a $2^{\circ} \mathrm{C}$. De igual manera, este mismo tratamiento, pone de manifiesto lo expresado en el análisis de correlaciones que indica que a mayor masa seca total, la calidad de flor aumenta, y la cosecha es más temprana.

Como ya se mencionó, las hojas en un inicio son el resultado de la conversión y translocación de carbohidratos de reservas a azúcares solubles desde el bulbo. Esta transformación es dependiente de la actividad de la enzima amilasa y ésta a su vez de la temperatura, pues el frío puede inducir la actividad de la $\alpha$-y $\beta$-amilasa, tal como se reporta para tubérculos de papa (Cochrane et al., 1991), por lo que los bulbos con mayor vernalización, tendrán mayor capacidad de enviar las reservas de carbohidratos para el desarrollo de la parte aérea.

Después de la siembra, la masa seca de los bulbos madre disminuye mientras que la masa de la parte aérea aumenta. Así mismo, la tasa de aumento y disminución de este parámetro es mayor cuando el tiempo de almacenamiento de los bulbos a bajas temperaturas aumenta; $y$ al expresar matemáticamente la variación de la masa seca durante el desarrollo de la planta, el modelo que mejor se ajusta es el exponencial (Inamoto et al., 2000). 


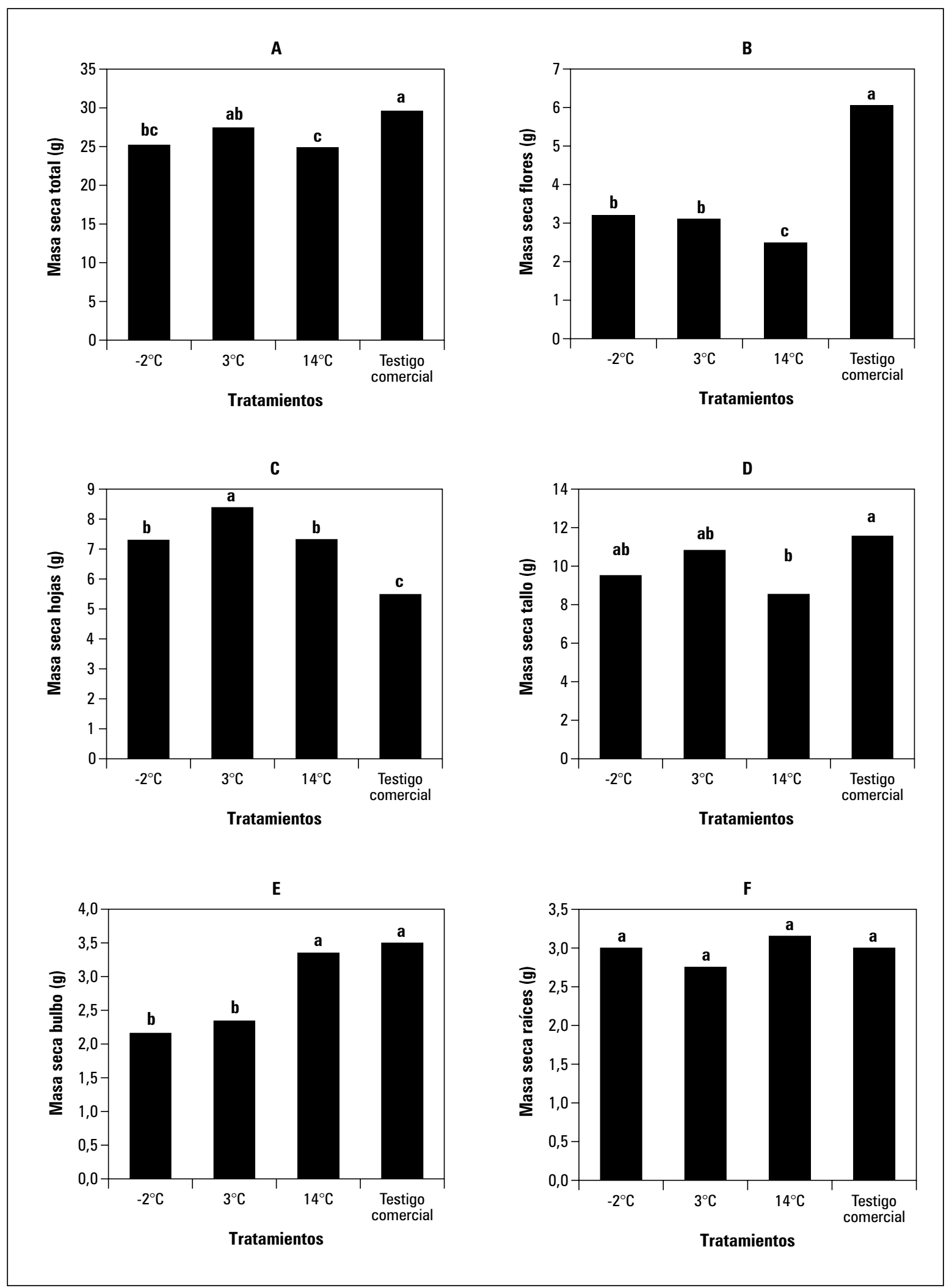

Figura 1. Efecto de la temperatura de vernalización sobre la distribución de masa seca en plantas de lirio provenientes de bulbos reutilizados. A. Total; B. Flores; C. Hojas; D. Tallo; E. Bulbo; F. Raíces. Promedios con letras distintas indican diferencia significativa según la prueba de Tukey $(P \leq 0,05)$. 
Tabla 2. Análisis de correlaciones de Pearson entre variables.

\begin{tabular}{|c|c|c|c|c|c|c|c|c|c|c|c|}
\hline $\begin{array}{l}\frac{\mathscr{d}}{0} \\
\frac{0}{\frac{10}{\frac{10}{50}}} \\
\frac{10}{>}\end{array}$ & 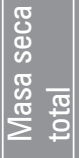 & 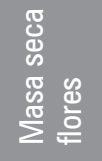 & 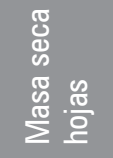 & 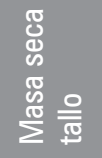 & 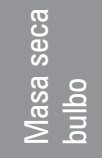 & 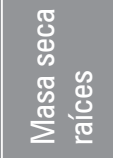 & 营 & 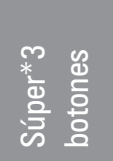 & 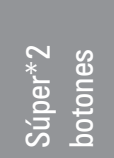 & 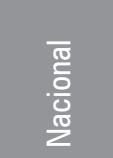 & $\begin{array}{l}\frac{\overline{0}}{\frac{E}{E}} \\
\frac{\bar{E}}{\text { C }}\end{array}$ \\
\hline Masa seca total & 1 & $0,71 * *$ & $-0,40 *$ & $0,88^{* *}$ & $0,3 \mathrm{~ns}$ & $-0,1 \mathrm{~ns}$ & $0,69 * *$ & 0,13 ns & $-0,5^{*}$ & $-0,34 \mathrm{~ns}$ & $-0,6^{* *}$ \\
\hline Masa seca flores & & 1 & $-0,68 * *$ & $0,41^{*}$ & $0,37 \mathrm{~ns}$ & $-0,02$ ns & $0,91 * *$ & $-0.03 n s$ & $-0,66 * *$ & $-0,48 *$ & $-0,6^{* *}$ \\
\hline Masa seca hojas & & & 1 & $-0,3 \mathrm{~ns}$ & $-0,6^{* *}$ & $-0,3 \mathrm{~ns}$ & $-0,65 * *$ & $0.18 \mathrm{~ns}$ & $0,57 * *$ & $0,36 \mathrm{~ns}$ & $0,21 \mathrm{~ns}$ \\
\hline Masa seca tallo & & & & 1 & $0,18 \mathrm{~ns}$ & $-0,2 \mathrm{~ns}$ & $0,48 *$ & $0,33 \mathrm{~ns}$ & $-0,3 \mathrm{~ns}$ & $-0,3 \mathrm{~ns}$ & $-0,6 * *$ \\
\hline Masa seca bulbo & & & & & 1 & $0,07 \mathrm{~ns}$ & $0,32 \mathrm{~ns}$ & $-0,46 *$ & $-0,5 * *$ & $0,02 \mathrm{~ns}$ & $0,22 \mathrm{~ns}$ \\
\hline Masa seca raíces & & & & & & 1 & $-0,1 \mathrm{~ns}$ & $-0,3 \mathrm{~ns}$ & $0,0 \mathrm{~ns}$ & $0,3 \mathrm{~ns}$ & $0,2 \mathrm{~ns}$ \\
\hline Extra & & & & & & & 1 & $0,0 \mathrm{~ns}$ & $-0,7 * *$ & $-0,6 * *$ & $-0,6^{* *}$ \\
\hline Súper*3 botones & & & & & & & & 1 & $-0,05 \mathrm{~ns}$ & $-0,3 \mathrm{~ns}$ & $-0,5^{* *}$ \\
\hline Súper* 2 botones & & & & & & & & & 1 & $0,23 \mathrm{~ns}$ & $0,3 \mathrm{~ns}$ \\
\hline Nacional & & & & & & & & & & 1 & $0,4 \mathrm{~ns}$ \\
\hline Sin flor & & & & & & & & & & & 1 \\
\hline
\end{tabular}

** Significancia al 1\%. * Significancia al 5\%. ns: no hay significancia según prueba de Pearson.

Según Moe (1979) y Moe y Wickstrom (1973), el frío afecta principalmente la conversión de almidón a azúcares solubles para el suministro de carbohidratos, induciendo una rápida brotación del bulbo, reduciendo así mismo el periodo de brotación a floración. La vernalización promueve la despolimerización de los carbohidratos, lo que resulta en un aumento de los azúcares solubles y los contenidos de nitrógeno soluble (Chouard, 1960). En concordancia, Rees (1966) también afirma que las bajas temperaturas promueven la conversión de almidón almacenado en azúcares solubles. Una acumulación de azúcares solubles y la glutamina en las escamas de los bulbos puede promover el rápido crecimiento del tallo y las hojas y la floración normal durante el cultivo (Ohyama et al., 1988). No obstante, es posible que esa degradación del almidón no se haya dado completamente en el tratamiento con menor vernalización $\left(14^{\circ} \mathrm{C}\right)$ y por eso la masa seca del bulbo al final fue mayor, de igual manera, este fenómeno trajo consigo que las plantas permanecieran en estado vegetativo y nuevamente se favoreciera más la masa seca del bulbo, pero con la consecuencia de que la floración en estas plantas fue escasa.
La mayor conversión de almidón a azúcares solubles se debe a la acción de las giberelinas (GA) cuya síntesis puede ser estimulada por bajas temperaturas, estas sintetizan enzimas hidrolíticas, principalmente $\alpha$-amilasa (Davies, 2004) isoamilasa, b-amilasa, a-glucosidasa y almidón fosforilasa (Cochrane et al., 1991; Cottrell et al., 1993; Claassen et al., 1993; Hill et al., 1996; Nielsen et al., 1997) encargadas de la degradación del almidón. Simultáneamente, los niveles de ABA disminuyen con el incremento en los niveles de frío y se ha encontrado que en bulbos de $L$. rubellum, almacenados a $4^{\circ} \mathrm{C}$, la concentración de este inhibidor del crecimiento disminuye a medida que la vernalización aumenta (Rong-Yan et al., 2006) y también coincide con los estudios en Polianthes tuberosa (Nagar, 1995) y Allium wakegi (Yamazaki et al., 2002). Lo anterior podría indicar que el ABA está implicado en la dormancia del bulbo, sin embargo, Rong-Yan et al. (2006) sugieren que en lirio, existen otros factores desconocidos que también están asociados con la inhibición de la brotación de los bulbos.

En tulipanes vernalizados a $5^{\circ} \mathrm{C}$ por un lapso de 2 meses se observó una rápida degradación 
del almidón después de iniciar el tratamiento y una considerable acumulación de sacarosa simultáneamente con la degradación. En bulbos no vernalizados, la conversión de almidón a azúcares solubles no se produjo antes de la plantación (Ohyama et al., 1988). Es posible que en los tratamientos de vernalización que no fueron lo suficientemente efectivos para convertir el almidón en azúcares solubles, la planta haya tenido la necesidad de enviar fotoasimilados al sistema radical y de esta manera aumentar la relación raíz/parte aérea, con el fin de que aumentara su envergadura para poder explorar el suelo y captar mayor cantidad de agua y nutrientes que suplieran las necesidades de la planta de lirio para su crecimiento.

Auzaque (2008) encontró una relación directa entre la masa de la flor y la vida en florero, esto muestra que una mayor cantidad de biomasa seca en los tejidos florales mantiene atrapada una buena cantidad de agua que mantiene turgente las flores, además, existe mayor sustrato respiratorio que garantiza una mayor vida poscosecha, por lo cual, se esperaría que las flores obtenidas con el testigo comercial y con la vernalización a $-2^{\circ} \mathrm{C}$ y $3^{\circ} \mathrm{C}$ tengan mayor vida poscosecha que las flores provenientes de los bulbos almacenados a $14^{\circ} \mathrm{C}$.

\section{Producción de flor}

Todas las calidades comerciales producidas de flor de lirio presentaron diferencias estadísticas (figura 2). El testigo comercial se caracterizó por un mayor porcentaje de calidad extra $(P \leq 0,01)$, el menor valor de calidad Súper*2 botones $(P \leq 0,01)$, no produjo calidad nacional $(P \leq 0,05)$ y la floración fue del $100 \%(P \leq 0,01)$ (figuras $2 \mathrm{~A}$, C, D y E). Por su parte, la vernalización artificial a $3^{\circ} \mathrm{C}$ generó la mayor cantidad de flores calidad Súper* 3 botones $(P \leq 0,01)$, Súper* 2 botones, así mismo bajo porcentaje de calidad nacional y de flores sin abrir (figuras $2 \mathrm{~B}, \mathrm{C}, \mathrm{D}$ y E). Los bulbos sometidos a $14^{\circ} \mathrm{C}$ presentaron la respuesta menos favorable, pues la producción de flores calidades Extra y Súper*3 botones fue la más baja, mientras que las flores de calidad nacional $y$ el porcentaje de flores sin abrir mostraron los mayores porcentajes (figuras $2 \mathrm{~A}, \mathrm{~B}, \mathrm{D}$ y E). En concordancia, Auzaque-Rodríguez et al. (2009) también encontraron $100 \%$ de floración con el testigo comercial, le siguió el tratamiento de los bulbos almacenados a $-2^{\circ} \mathrm{C}$ durante 8 semanas, mientras que el menor porcentaje se logró en los bulbos a $14^{\circ} \mathrm{C}$ sin importar el tiempo de vernalización.

En los tratamientos de vernalización donde el porcentaje de floración fue alto, posiblemente hubo regulación epigenética, que requiere de un proceso metabólico activo que garantice el proceso de división celular y replicación del DNA (Thomáshow, 2001; Lee y Amasino, 1995). Probablemente en lirio, al igual que en los ecotipos de invierno anual de Arabidopsis se requiere de vernalización y de días largos para florecer, pues en esta especie se identificó un gen que actúa como un represor de la floración denominado: FLOWERING LOCUS C (FLC). Este es altamente expresado en meristemos apicales sin vernalización (Dennis et al., 2006; Sung et al., 2006; Michaels y Amasino 2000), pero posterior a la vernalización, este gen es epigenéticamente reprimido (Michaels y Amasino, 1999; Sheldon et al., 1999) por un mecanismo desconocido por el resto del ciclo de vida de la planta, pero se conoce que induce cambios en la estructura de la cromatina de este gen represor (Sung y Amasino, 2004b) permitiendo florecer en respuesta a los días largos. Sin embargo, en la siguiente generación, el gen se vuelve a expresar, restaurando el requerimiento de frío. Así, en Arabidopsis el estado de expresión del gen FLC representa una mayor capacidad de competencia del meristemo (Michaels y Amasino, 2000).

Además, es posible que con la vernalización de los bulbos reutilizados a $-2^{\circ} \mathrm{C}$ y $3^{\circ} \mathrm{C}$ se haya garantizado la síntesis de suficiente concentración de giberelinas que sustituyen la necesidad de días largos después del periodo de frío (Salisbury y Ross, 1994) para que el lirio pueda florecer y lo 
A

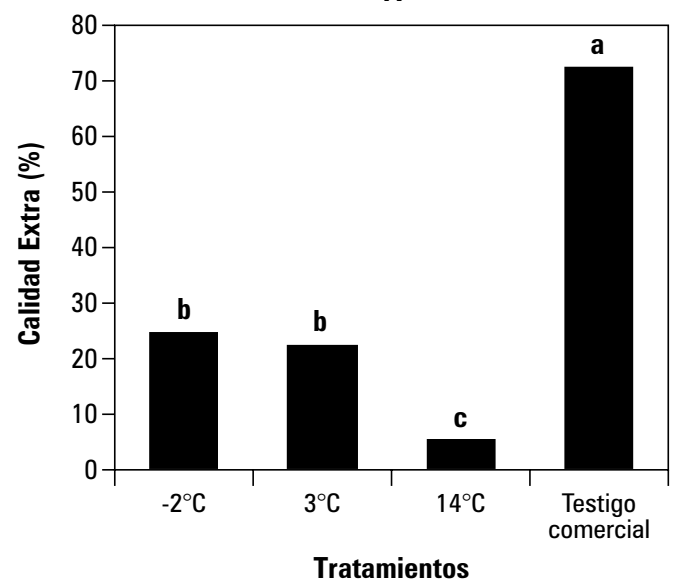

C

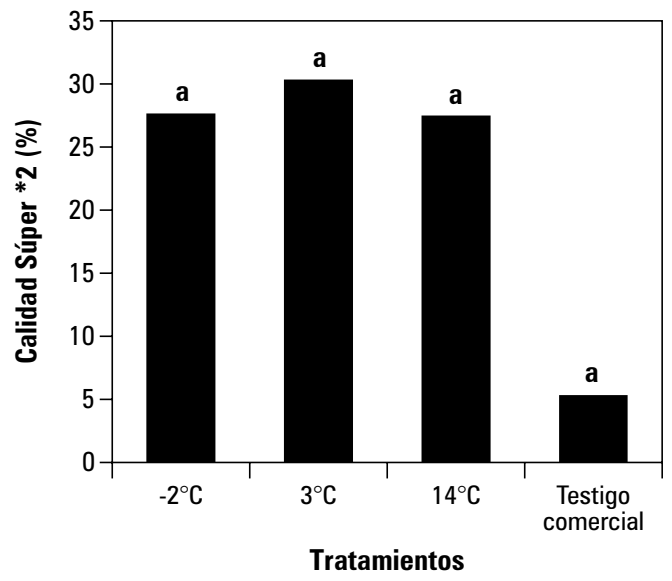

E

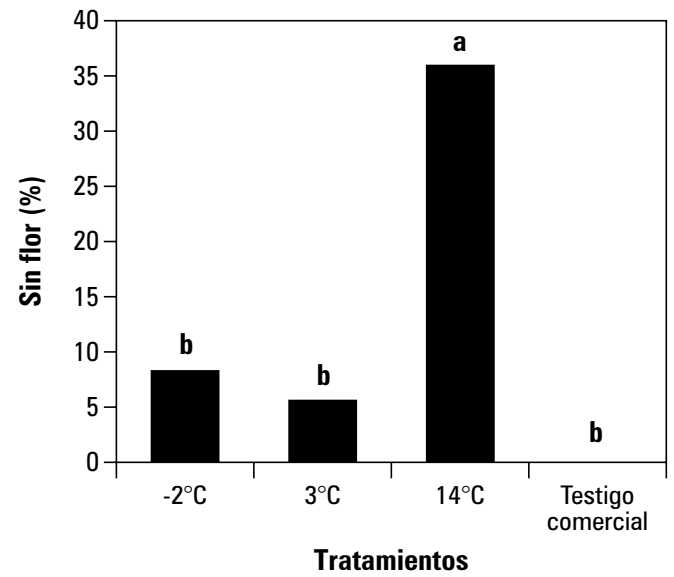

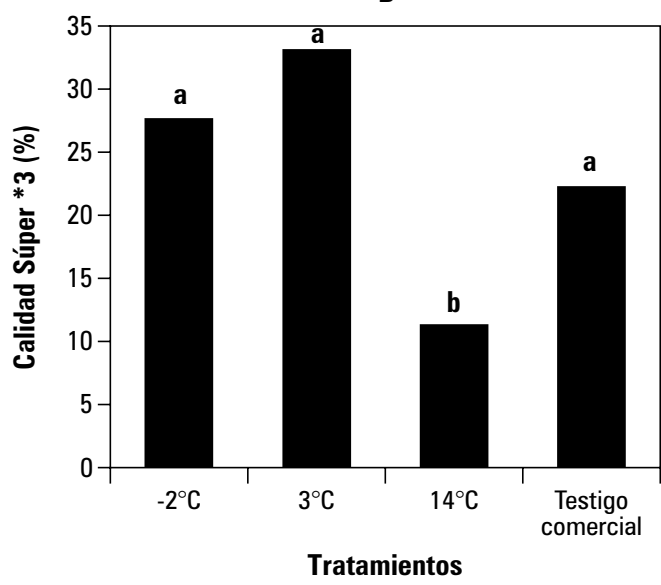

D

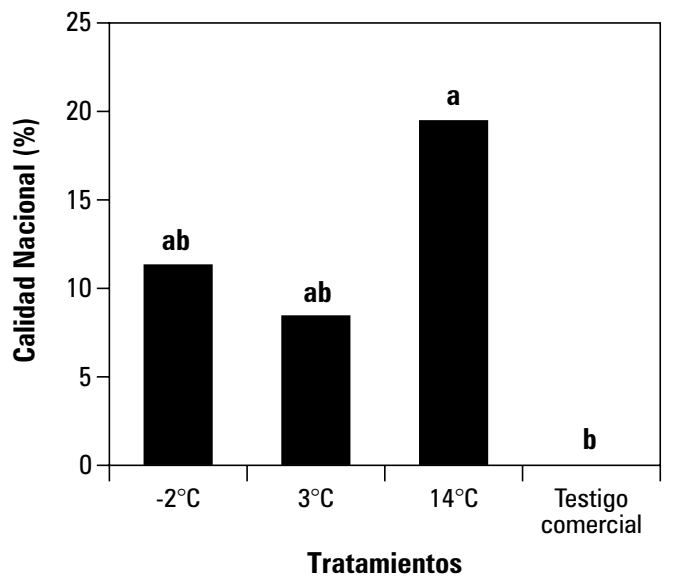

Figura 2. Efecto de la temperatura de vernalización sobre las calidades comerciales de flor de lirio provenientes de bulbos reutilizados. A. Calidad Extra; B. Calidad Súper *3 botones; C. Calidad Súper * 2 botones; D. Calidad Nacional; $E$. Sin flor. Promedios con letras distintas indican diferencia significativa según la prueba de Tukey $(P \leq 0,05)$. 
haga en mayor porcentaje y en un menor tiempo. Resultados similares se obtuvieron en Allium cepa, en donde tratamientos con frío a $5^{\circ} \mathrm{C}$ aumentaron el porcentaje de florecimiento, comparado con el control (Reghin et al., 2005).

Las inflorescencias del lirio consisten en varias yemas que difieren en el estado de desarrollo, y muchas de estas permanecen inmaduras en el momento de la cosecha. La formación de flores maduras depende del suministro de carbohidratos (Van Der Meulen-Muisers et al., 2001). Antes de la cosecha, las inflorescencias, se suplen con carbohidratos de la fotosíntesis. Después de alcanzar la inflorescencia estos asimilados se distribuyen entre las distintas yemas florales. La proporción de asimilados absorbidos por cada yema depende o está correlacionada en gran medida por la capacidad de síntesis de estos productos por parte de los órganos fuente (Ho, 1988).

Según Wang y Breen (1986), en Lilium las yemas florales expresan una alta dependencia de los órganos fuente, probablemente correlacionada con el incremento en el crecimiento de los tejidos florales (Clement et al., 1996). Esto explica la correlación entre la masa seca de flores y de hojas (-0,68; tabla 2), que además indica que existe translocación de fotoasimilados de las hojas a las flores para que garanticen su normal desarrollo. De otra parte, las correlaciones encontradas entre la masa seca de flores y las diferentes calidades indican que a media que la calidad comercial disminuye, la masa seca de estas flores es menor (tabla 2). También se observa que producir flores de calidad extra genera disminución de las demás calidades comercial, excepto Súper*3 botones. Al respecto, AuzaqueRodríguez et al. (2009) mencionan que lo recomendable es lograr obtener altos porcentajes del grado Extra y bajos porcentajes del grado nacional y Súper*2 botones.

Consecuentemente, el estado de madurez de las yemas es marcado por el contenido de carbohidratos en los tépalos, ya que las yemas más maduras contienen más carbohidratos (Van Der Maulen-Muisers et al., 2001) y la falta de apertura de las yemas de lirio podría estar asociada a la falta de carbohidratos como se ha encontrado en inflorescencias de otras especies bulbosas (Spikman, 1989). Los bulbos reutilizados que se almacenaron a $14^{\circ} \mathrm{C}$ presentaron la mayor cantidad de plantas con tallos sin flor, lo que pone de manifiesto la importancia de la vernalización para inducir la floración en lirio y es reportado por otros autores (Auzaque-Rodríguez et al., 2009; Taiz y Zeiger, 2006; Wilson y Dean, 1996).

\section{CONCLUSIONES}

Los bulbos importados generaron mayor floración y calidad de flor de lirio, además, presentaron mayor masa seca total, de flores, tallo y bulbo. Los bulbos reutilizados y vernalizados a $-2^{\circ} \mathrm{C}$ y $3^{\circ} \mathrm{C}$ también mostraron alto porcentaje de floración y buena calidad de flor. Los bulbos sometidos a $14^{\circ} \mathrm{C}$ tienen baja floración y calidad de flor.

\section{AGRADECIMIENTOS}

Los autores expresamos nuestros más sinceros agradecimientos al Ing. Agr., M.Sc. Orlando Auzaque-Rodríguez (qepd), quien dedicó su vida a la floricultura y en especial a la investigación y producción de flor de lirio en Colombia, uno de sus trabajos más importantes fue "El efecto de la vernalización de bulbos reutilizados sobre la calidad de la flor de lirio en la sabana de Bogotá", del cual se han derivado varias investigaciones. Orlando, paz en tu tumba, te recordaremos por siempre.

Este trabajo se realizó con apoyo de Colciencias y la Universidad Pedagógica y Tecnológica de Colombia (UPTC) mediante el programa nacional de formación de investigadores "Generación del bicentenario" programa jóvenes investigadores e innovadores "Virginia Gutiérrez de Pineda" del cual fue merecedor Helber Enrique Balaguera-López. 


\section{REFERENCIAS BIBLIOGRÁFICAS}

Alberto, C., N.A. Streck, L.C. Walter, H. Telles, A. Brackmann, F. Oliveira, A. Zanon y L. Fagundes. 2009. Resposta à vernalização de cultivares brasileiras de trigo. Bragantia 68(2), 535-54.3

Auzaque-Rodríguez, O., H.E. Balaguera-López, J.G. Álvarez-Herrera y G. Fischer. 2009. Efecto de la vernalización de bulbos reutilizados sobre la calidad de la flor de lirio (Lilium sp.) en la Sabana de Bogotá. Agron. Colomb. 27(1), 65-71.

Auzaque, O. 2008. Efecto de la vernalización de bulbos sobre la calidad de la flor de lirio (Lilium sp.) en la Sabana de Bogotá. Tesis de maestría. Facultad de Agronomía, Universidad Nacional de Colombia, Bogotá.

Azcón-Bieto, J. y M. Talón. 2008. Fundamentos de fisiología vegetal. $2^{a}$. ed. McGraw Hill Interamericana de España, Madrid.

Chouard, P. 1960. Vernalization and its relations to dormancy. Annu. Rev. Plant Physiol. 11, 191-238.

Claassen, P.A.M., M.A.W. Budde y M.H. Van Calker. 1993. Increase in phosphorylase activity during coldinduced sugar accumulation in potato tubers. Potato Res. 36, 205-217.

Clement, C., M. Burrus y J.C. Audran. 1996. Floral organ growth and carbohydrate content during pollen development in Lilium. Amer. J. Bot. 83, 459-469.

Cochrane, M.P., C.M. Duffers, M.J. Allison y G.R. Mackay. 1991. Amylolytic activity in stored potato tubers. 2. The effect of low temperature storage on the activities of $\mathrm{a}$ - and $\mathrm{b}$-amylase and a-glucosidase in potato tubers. Potato Res. 34, 333-341.

Cottrell, J.E., C.M. Duffers, L. Paterson, G.R. Mackay, M.J. Allison y H. Bain. 1993. The effect of storage temperature on reducing sugar concentration and the activities of three amylolytic enzymes in tubers of the cultivated potato, Solanum tuberosum L. Potato Res. 36, 107-117.

Davies, P.J. 2004. Plant hormones - biosynthesis, signal transduction, action! $3^{\text {rd }}$ ed. revised. Springer, Berlin

De Hertogh A. 1996. Marketing and research requirements for Lilium in North America. Acta Hort. 414, $17-24$.

Dennis, E., C. Helliwell y W. Peacock. 2006. Vernalization: spring into flowering. Dev. Cell 11, 1-7.
Hill, L., R. Reimholz, R. Schroder, T.H. Nielsen y M. Stitt. 1996. The onset of sucrose accumulation in cold stored potato tubers is caused by an increased rate of sucrose synthesis and coincides with low levels of hexose phosphates, an activation of sucrose phosphate synthase and the appearance of a new form of amylase. Plant Cell Environ. 19, 1223-1237.

Ho, L. 1988. Metabolism and compartmentation of imported sugars in sink organs in relation to sink strength. Annu. Rev. Plant Physiol. Plant Mol. Biol. 39, 355-378.

Inamoto, K., T. Hase, M. Doi y H. Imanishi. 2000. Effects of duration of bulb chilling on dry matter distribution in hydroponically forced tulips. Sci. Hortic. 85, 295-306.

Kim, S.H., C.E. Niedziela, P.V. Nelson, A.A. De Hertogh, N.C. Mingis y W.H. Swallow. 2007. Growth and development of Lilium longiflorum 'Nellie White' during bulb production under controlled environments. I. Effects of costant, variable and greenhouse day/night temperature regimes on scale and stem bulblets. Sci. Hortic. 112, 89-94.

Klock, P. 1997. Flores de plantas bulbosas. Ed. Everest, León, España.

Lee, S.J., M.S. Roh. 2001. Influence of frozen storage duration and forcing temperature on flowering of oriental hybrid lilies. HortScience 36(6), 1053-1056.

Lee, S.J., Y.A. Kim y H.J. Wang. 1996. Effect of bulb vernalization on the growth and flowering of asiatic hybrid lily. Acta Hort. 414, 229-234.

Lee, I. y R.M. Amasino 1995. Effect of vernalization, photoperiod and light quality on the flowering phenotype of Arabidopsis plants containing the FRIGIDA gene. Plant Physiol. 108,157-162.

Michaels, S.D. y R.M. Amasino. 2000. Memories of winter: vernalization and the competence to flower. Plant Cell Environ. 23, 1145-1153.

Michaels, S.D. y R.M. Amasino, 1999. Flowering locus $\mathrm{C}$ encodes a novel MADS domain protein that acts as a repressor of flowering. Plant Cell 11, 949-956.

Moe, R. 1979. Effect of precoolong at 5 or $-1^{\circ} \mathrm{C}$ on shoot growth, flowering, and carbohydrate metabolism in tulip bulbs. Sci. Hortic. 10, 187-201. 
Moe, R. y A. Wickstrom. 1973. The effect of storage temperature on shoot growth, flowering, and carbohydrate metabolism in tulip tulbs. Physiol. Plant. 28, 81-87.

Nagar, P.K. 1995. Changes in abscisic acid, phenols and indoleacetic acid in bulbs of tuberose (Polianthes tuberosa L.) during dormancy and sprouting. Sci. Hortic. 63, 77-82.

Nielsen, T.H., U. Deiting y M. Stitt. 1997. A b-amylase in potato tubers is induced by storage at low temperature. Plant Physiol. 113, 503-510.

Ohyama, T., T. Ikarashi y A. Baba, 1988. Effect of cold storage treatment for forcing bulbs on the $\mathrm{C}$ and $\mathrm{N}$ metabolism of tulip plants. Soil Sci. Plant Nutr. 34(4), 519-533.

Rees, A.R. 1966. The physiology of ornamental bulbous plants. Bot. Rev. 32(1), 1-23.

Reghin, M.Y., R.F. Otto, J.R. Olinik, C.F.S. Jacoby y R.P. Oliveira. 2005. Vernalização em bulbos e efeito no rendimento e potencial fisiológico de sementes de cebola. Hort. Brasil. 23(2), 294-298.

Rong-Yan, X., N. Yoshiji y H. Dong-Sheng. 2006. Changes in endogenous abscisic acid and soluble sugars levels during dormancy-release in bulbs of Lilium rubellum. Sci. Hortic. 111, 68-72.

Salisbury, F.B. y C.W. Ross. 1994. Fisiología vegetal. Grupo Editorial Iberoamérica S.A., México.

Sheldon C.C., J.E. Burn, P.P. Perez, J. Metzger, J.A. Edwards, W.J. Peacock y E.S. Dennis. 1999. The FLF MADS box gene: a repressor of flowering in Arabidopsis regulated by vernalization and methylation. Plant Cell 11, 445-458.

Spikman, G. 1989. Development and ethylene production of buds and florets of cut freesia inflorescences as influenced by silver thiosulphate, aminoethoxyvinylglucine and sucrose. Sci. Hortic. 39, 73-81.

Streck, N. A. y M. Schuh. 2005. Simulating the vernalization response of the 'Snow Queen' lily (Lilium longiflorum Thunb.) Sci. Agric. 62(2), 58-64.
Streck, N.A. 2002. A generalized vernalization response function for lily (Lilium spp.). Rev. Brasil. Agrometeor. 10(2),221-228.

Sung, S., H.E.Y. Eshoo, T.W. Tamada L., Johnson, K. Nakahigashi K. Goto, S.E. Jacobsen y R.M. Amasino. 2006. Epigenetic maintenance of the vernalized state in Arabidopsis thaliana requires LIKE HETEROCHROMATIN PROTEIN 1. Nat. Genet. 38, 706-710.

Sung, S. y R.M. Amasino. 2004a. Vernalization and epigenetics: how plants remember winter. Current Opinion Plant Biol. 7, 4-10.

Sung, S. y R.M. Amasino. 2004b.Vernalization in Arabidopsis thaliana is mediated by the PHD finger protein VIN3. Nature 427, 159-164.

Taiz, L. y E. Zeiger. 2006. Plant physiology. $3^{\text {rd }}$ ed. Sinauer Associates Inc. Publishers, Sunderland, MA.

Thomashow, M.F. 1999. Plant cold acclimation: freezing tolerance genes and regulatory mechanisms. Annu. Rev. Plant. Physiol. Plant. Mol. Biol. 50, 571-599.

Van Der Meulen-Muisers, J.M., J.C. Van Oeveren, H. Linus, W. Van Der Plas y J. M. Van Tuyl. 2001. Postharvest flower development in Asiatic hybrid lilies as related to tepal carbohydrate status. Postharvest Biol. Technol. 21, 201-211.

Wang, Y.T. y P.J. Breen, 1986. Partitioning of 14C-assimilate in Easter Lily as affected by growth stage and flower removal. Sci. Hortic. 29, 273-281.

Wilson, A. y C. Dean. 1996. Analysis of the molecular basis of vernalization in Arabidopsis thaliana. Cell Develop. Biol. 7, 435-440.

Xu, R., Y. Niimi y D. Han. 2006. Changes in endogenous abscisic acid and soluble sugars levels during dormancy-release in bulbs of Lilium rubellum. Sci. Hortic. 111(1), 68-72.

Yamazaki, H., T. Nishijima, M. Koshioka y H. Miura. 2002. Gibberellins do not act against abscisic acid in the regulation of bulb dormancy of Allium wakegi Araki. Plant Growth Regul. 36, 223-229. 